\title{
Enterococcus gallinarum
}

National Cancer Institute

\section{Source}

National Cancer Institute. Enterococcus gallinarum. NCI Thesaurus. Code C86370.

A species of facultatively anaerobic, Gram-positive, cocci shaped bacteria in the phylum Firmicutes. This species is nonmotile, non-spore forming, catalase negative, does not hydrolyze gelatin, does not produce hydrogen sulfide and is esculin and hippurate positive. E. gallinarum has been isolated from the gastrointestinal tract of domestic fowl and is pathogenic in humans with some resistance to vancomycin. 